\title{
10
}

Saris D

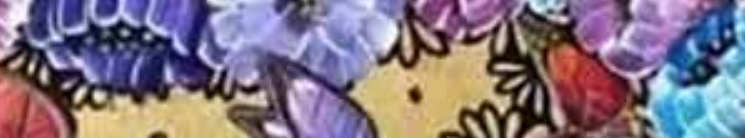

$\cos x^{2}$

Sin

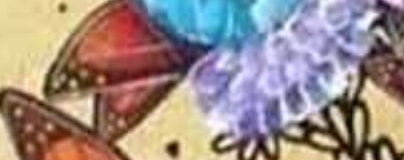

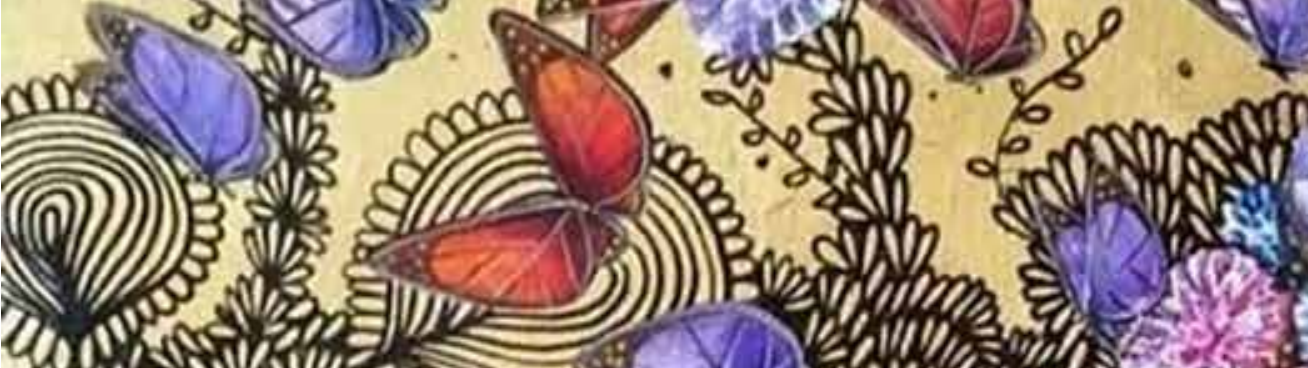

bognus

(

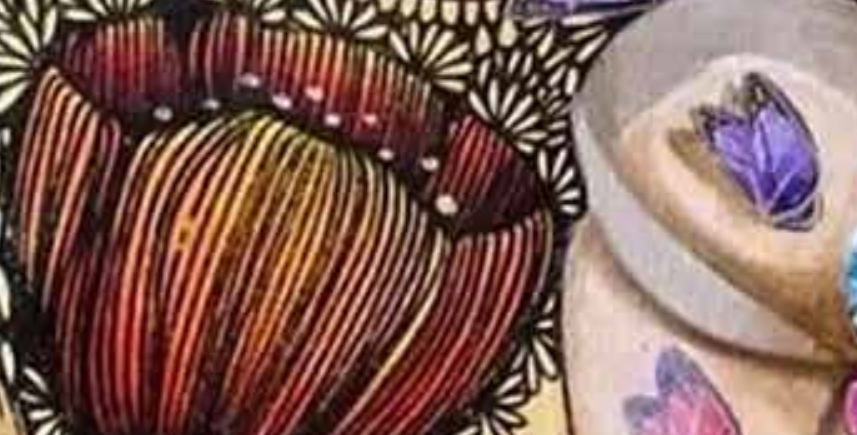

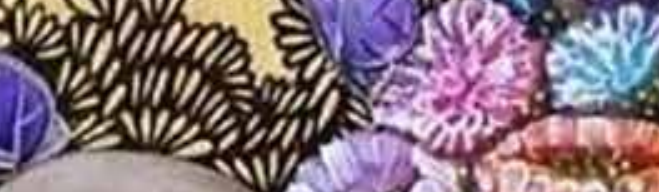

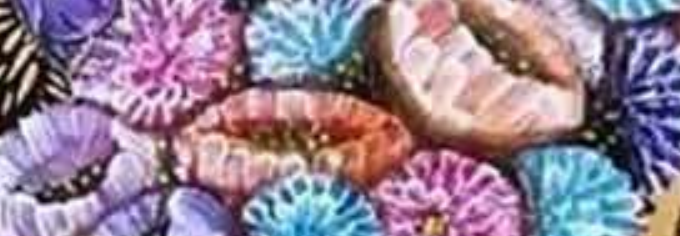

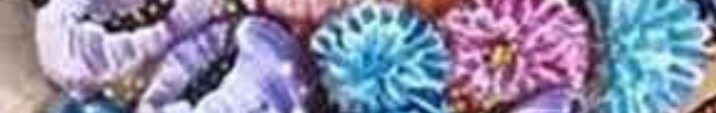

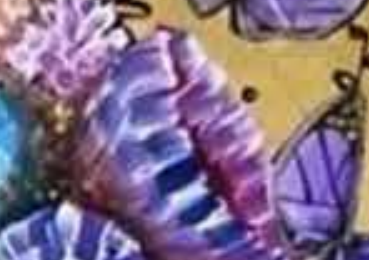
rat

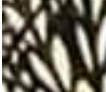

Nitiv

(n).

N.

8

Nitas

10.1.

11

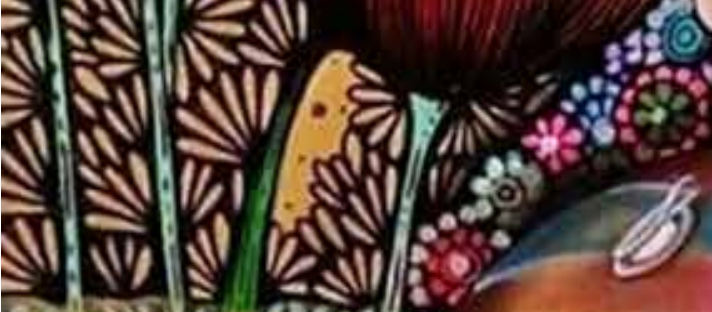

WI $\frac{}{\mathrm{N}}$

Now?

(1)

a.

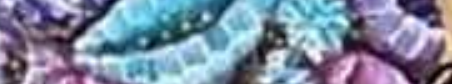

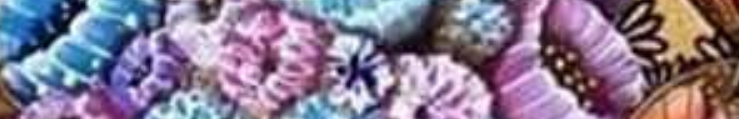

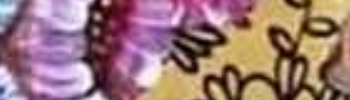

- 1 s.

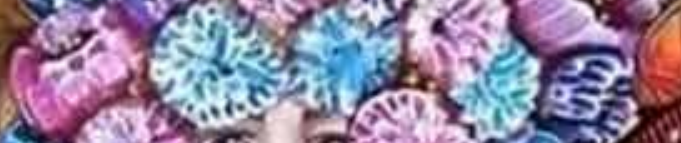

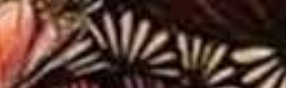

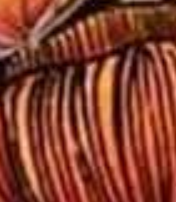

1) 


\section{LA MUJER}

Con manos trabajadoras construyendo va, los nidos, desterrando la pobreza, alimentando a sus hijos.

El reloj no marca horas, trabaja para su casa, se entrega en cuerpo y alma, a favor de los que ama.

Tiene valor, fortaleza, es guerrera y es soldado, contra tormentas pelea, protegiendo a sus hermanos.

Fabricante de esperanzas, elevándose hasta el cielo, la mujer por sus valores, es quien mueve el universo.

Arjona Delia 


\title{
7. Determinantes sociodemográficos de la participación femenina en el mercado laboral de Honduras, años 2011 y 2015
}

\author{
María del Carmen Torres Marquina ${ }^{1}$ \\ DOI: https://doi.org/10.5377/pdac.v16i0.10231 \\ Recibido: 20/04/2020 - Aceptado: 26/05/2020
}

\begin{abstract}
Resumen: El presente artículo tiene como objetivo general analizar las variables sociodemográficas que determinan la participación laboral de la mujer para los años 2011 y 2015. Se estimaron modelos de tipo probit para identificar las variables con mayor o menor impacto en la probabilidad de que la mujer participe en el mercado laboral en Honduras.

Se concluye que el nivel educativo influye positivamente para que la mujer se inserte en el mercado laboral obteniendo empleos e ingresos dignos, así pues, se constató que las mujeres con nivel de secundaria o superior, estaban laborando en ocupaciones de mejor jerarquía, mientras que las mujeres con nivel primaria se insertan en ocupaciones de baja categoría, y eran las mujeres con educación superior las que obtuvieron los ingresos más altos.

Las mujeres solteras, son las que en un mayor porcentaje se encuentran insertas en el mercado de trabajo (46\%), respecto a las casadas o en unión libre ( $45 \%$ entre ambas). El área de residencia rural, influye negativamente, se identificaron los mayores porcentajes de mujeres trabajando en el área urbana; mientras que el número de hijos menores proporcionó evidencia negativa para que la mujer obtenga un empleo.
\end{abstract}

Palabras claves: mercado laboral, población femenina económicamente activa, modelo probit.

Sociodemographic determinants of women's participation in the labor market of Honduras, 2011 and 2015

Abstract: The objective of this article is to analyze the sociodemographic variables that determine women's labor participation for the years 2011 and 2015. Probit-type models were estimated to identify the variables with greater or less impact on the probability that women participate in the labor market in Honduras.

Concluding that the educational level positively influences the insertion of women into the labor market, obtaining decent jobs and income, therefore, it was found that women with a secondary or higher level of education were working in higher-ranking occupations, while women at the primary level of eductarion are inserted in low-category occupations, and it was women with higher education who obtained the highest income.

Unmarried women are who, in a higher percentage, are inserted in the labor market (46\%), compared with the married or in a consensual union ( $45 \%$ between the two). The rural residence area negatively influences; the highest percentages of women working were identified in the urban area; while the number of children provided negative evidence for a woman to obtain a job.

Keywords: labor market, economically active female population, probit model.

\section{Introducción}

Históricamente la mujer ha reflejado una baja participación en el mercado laboral; no solo en Honduras o países en vías de desarrollo, sino incluso, en países con economías y sociedades más avanzadas. Muchas teorías tratan de fundamentar este hecho, desde el machismo cultural hasta la explicación de una mayor productividad de la mujer en tareas del hogar con respecto al hombre; pues se dice que la mujer sería más productiva en tareas del hogar, cuidando de este y de los hijos. Las diferencias de género en el mercado laboral se ven como un hecho normal, sin embargo, a la larga esto genera disparidad en las oportunidades de desarrollo humano para las mujeres (Becker, 1971, p. 15).

Durante los últimos 20 años, la tasa de participación femenina ha venido generando una reducción en la brecha respecto a la de los hombres. Algunos de los argumentos de por qué es menor la participación de la mujer en la actividad económica, están relacionados con aspectos tales como: el matrimonio, embarazos a temprana edad, número de hijos, el nivel educativo, la subordinación histórica, la dependencia económica de las mujeres y la no aplicación de políticas públicas relacionadas con la equidad y garantías de los derechos laborales de la mujer.

1 Máster en Demografía y Desarrollo UNAH, Docente de la Maestría en Demografía y Desarrollo, UNAH. e-mail: mariadel.torres@unah.edu.hn 
En el caso de las mujeres casadas/unidas con hijos, la decisión de incorporarse al mercado de trabajo puede ser compleja debido a que ésta tiene que evaluar si el trabajo le va generar más beneficios. La mujer al insertarse en el mercado laboral debe combinar su papel de madre y responsable del cuidado diario del hogar y la educación de los hijos; con la responsabilidad de traer recursos adicionales o suficientes para la sobrevivencia de la familia, lo que aumenta su vulnerabilidad al momento de acceder al mercado laboral; esta situación hace que se inserte en trabajos informales, mal remunerados o de poca categoría. Sin embargo, y pese a que hay un subregistro en las estadísticas del país, éstas han proporcionado evidencia de que la mujer participa cada vez más en el mercado laboral; aún cuando todavía los niveles de dicha participación siguen siendo menor en relación al porcentaje masculino.

Si bien es cierto que la participación de la mujer en el mercado de trabajo ha ido en aumento, vale la pena preguntarse: ¿En qué medida, se debe a sus logros educativos? o ¿En qué medida la decisión de tener menos hijos ha incidido en la inserción de la mujer en el mercado laboral? Estás serán las principales interrogantes que se desarrollan en el presente artículo.

\section{Metodología}

La presente investigación desarrolló una caracterización de variables demográficas y económicas de las mujeres; de igual manera se desarrollaron modelos probit, para dar respuesta a la hipótesis propuesta en el estudio la cual es: "Los factores que inciden en la participación laboral de la mujer hondureña son: hijos menores de 7 años, el estado civil (casado), área de residencia, nivel educativo, la edad y la jefatura de hogar" se trata de identificar cuáles de estas variables son significativas en la probabilidad de participación de la mujer en el mercado de trabajo.

La función probit se denomina de la siguiente manera:

\section{Dónde:}

$$
\operatorname{Pr}(\mathrm{Y}=1 \mid \mathrm{X})=\Phi(\mathrm{XT} \beta)
$$

Pr: denota la probabilidad del evento.

$Y$ : es la variable dependiente.

$X$ : es la variable explicativa o independiente.

$\Phi$ : es la función de distribución acumulada de la distribución normal estándar.

Los parámetros $\beta$ se estiman típicamente por máxima verosimilitud.
Los resultados obtenidos del Modelo Probit Multivariado, estimado para las mujeres hondureñas de la PEA (10 a 64 años), incluyó como variable dependiente: inserción laboral y las variables explicativas o independientes son: hijos menores de 7 años, estado civil (casado), área de residencia (rural), mujer jefa de hogar y nivel educativo.

- Variable dependiente: la participación de la mujer en el mercado laboral (PEA femenina), la cual toma valores 1 trabaja, 0 no trabaja.

- Variables independientes: hijos menores 7 (tothijos); estado civil (casado), área de residencia rural (rurur2), jefa de hogar (jefemuj), nivel educativo (edu1, edu2, edu3, edu4) sin nivel, primaria, secundaria, superior respectivamente.

Las variables que resultaron significativas al 95\% de nivel de confianza en el modelo, fueron:

- Variable dependiente $=$ trab_1 (trabaja: 1,0$)$.

- Variables independientes = tothijos (que son los hijos menores de 7 años por mujer), casado, rurur2 (área rural), jefemuj (jefe mujer), edu2 (primaria), edu3 (secundaria), edu4 (superior).

Para el desarrollo del estudio se utilizó bases de datos en SPSS consolidadas (4 trimestres) de las Encuestas de Hogares de Propósitos Múltiples del Instituto Nacional de Estadística (INE), para los años 2011 y 2015, la muestra para estas bases de datos es de alrededor 80,000 registros en promedio, lo que proporciona una mayor robustez en la estimación de los indicadores; así también, para el desarrollo de los modelos probit se utilizó la herramienta para análisis estadístico STATA 15.

La investigación fue concebida de tipo relacional explicativo, en vista que se estudia las relaciones entre variables dependientes e independientes, así mismo de tipo explicativo ya que se busca estudiar el porqué del comportamiento de algunas variables estableciendo relaciones de asociación entre ellas, mediante la descripción y cuantificación de sus tendencias.

Los cambios demográficos en la población pueden exigir de los gobiernos, demandas sociales, en ese sentido, este estudio adquiere relevancia, ya que tiene estrecha relación con la formulación, creación y ejecución de políticas laborales, las cuales deben ser integrales para satisfacer las necesidades requeridas por la población femenina, por ello la importancia de éste, ya que podría abrir un camino hacia el conocimiento científico ligado a la relación entre 
población y mercado laboral, especialmente para las mujeres.

\section{Discusión de resultados}

En el contexto de las estadísticas nacionales presentadas se plantea como pregunta central de la investigación la siguiente: ¿Qué factores sociodemográficos determinan la participación de la mujer en el mercado laboral hondureño?

Se caracteriza la PEA femenina desde el aspecto sociodemográfico y económico para luego desarrollar modelos probit multivariado y examinar las variables que determinan o inciden en la probabilidad de participación laboral femenina.

\subsection{Características sociodemográficas de la PEA femenina}

3.1.1. Estructura de la PEA femenina por jefatura de hogar según edad

En el año 2011 el número de hogares a nivel nacional ascendía a 1,737,385 de los cuales el 33.4\% $\left(580,891\right.$ hogares) eran liderados por mujeres ${ }^{2}$, mientras que para 2015 el número total de hogares a nivel nacional fue 1,929,667 de los cuales $33.3 \%$ $(642,289$ hogares) eran jefes mujeres.

La PEA femenina jefe de hogar en 2011 era de $54 \%$; y para 2015 alcanza 56\%; esto quiere decir que aproximadamente 6 de cada 10 mujeres jefes de hogar son parte de la PEA, el resto está calificada como inactiva.

Al analizar la distribución por edades quinquenales para 2011 se observa que el mayor porcentaje de la PEA femenina jefe de hogar, se encuentra entre las edades de 35-39 años (13.8\%), para 2015 este porcentaje es de $13.7 \%$ y corresponde a las edades de 40-44 años. Los porcentajes más bajos de la PEA femenina corresponde a las edades de 15-18; 0.3\% y $0.2 \%$ respectivamente (Gráfico $\mathrm{N}^{\circ} 1$ ).

Según Clarck (2000), en los estudios sobre el impacto del trabajo (conductual y emocional) sobre la familia, se ha reconocido que una de las condiciones subjetivas de la calidad de vida de los trabajadores radica en la interacción de los ámbitos familiar y laboral, reconociéndose que ambos se influyen recíprocamente (Casas, Repullo, Lorenzo y Cañas, 2002, p. 87).

\subsubsection{Estructura de la PEA femenina por hijos me- nores de 7 años}

En general existe una marcada desigualdad de género en el trabajo asociada a la maternidad, pues con esta, se espera que las mujeres se ocupen de los hijos, reduciendo su participación laboral o no trabajando más. Un determinante que condiciona la inserción de la mujer en el mercado laboral, es la presencia de hijos pequeños, sobre todo cuando

\section{Gráfico №1 PEA femenina por jefatura de hogar según edad, años 2011 y 2015}

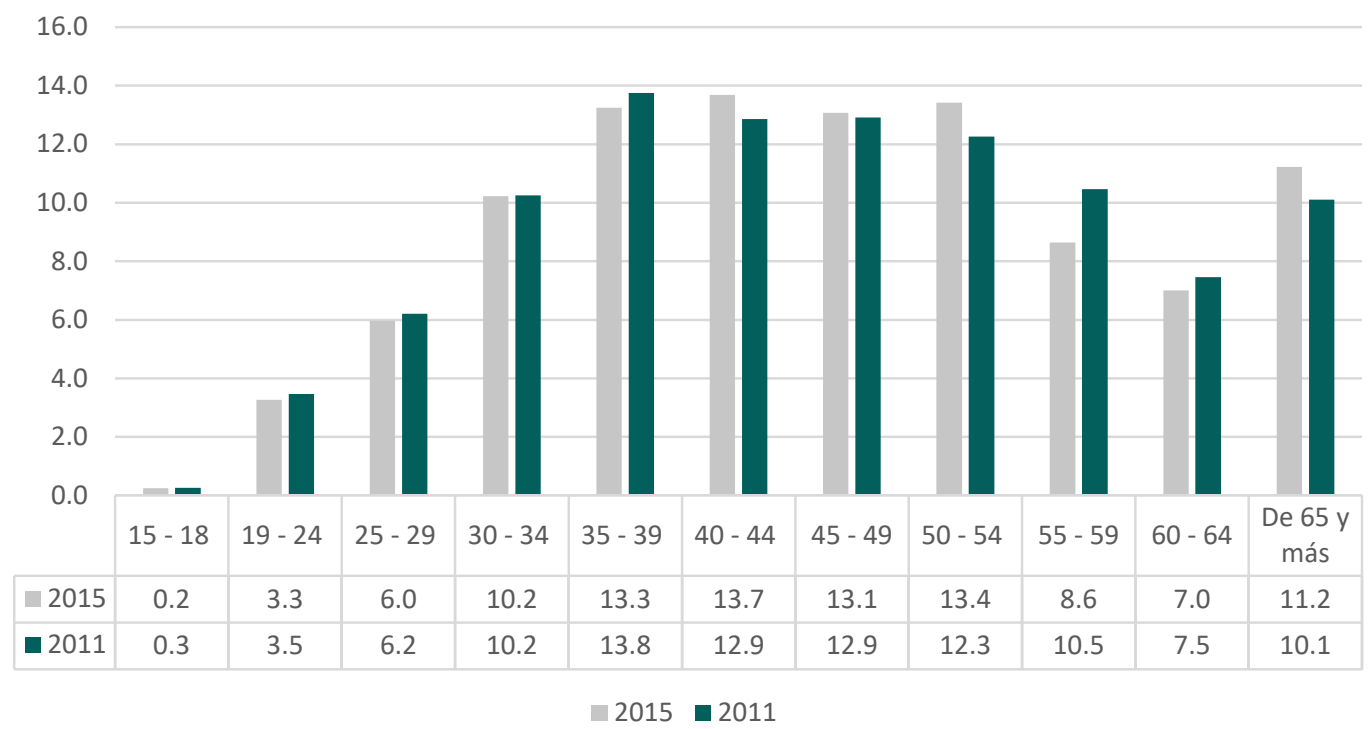

Fuente: elaboración propia en base a EPHPM, 2011 y 2015, INE.

2 Cabe señalar que en Honduras la jefatura de hogar femenina se toma a partir de los 15 años. 
éstos aún no tienen edad para asistir a la educación formal, pues dependen en mayor porcentaje de los cuidados de su madre; es por esta razón que se ha considerado en esta estimación tomar los hijos menores de 7 años.

Al analizar el Gráfico $\mathrm{N}^{\circ} 2$, se estimó un total de 384,259 hijos para la población femenina económicamente activa en 2011 y para 2015 este número ascendió a 521,800 hijos menores de 7 años. Analizando el número de hijos según el nivel educativo de la madre; se observa que aproximadamente en el $50 \%$ de los casos, la madre contaba con nivel de educación primaria en ambos años. En el caso de las madres que tenían educación secundaria el valor promedio fue $35 \%$ para ambos años; y apenas un $9 \%$ en 2011 contaba con educación superior valor que aumenta a $11 \%$ en 2015 . El porcentaje más bajo corresponde a las mujeres sin nivel educativo $6 \%$ y $4.9 \%$ para cada año respectivamente.

En general la PEA femenina con hijos menores contaba con nivel de educación primaria, siendo esta condición una desventaja para las madres; pues en teoría las mujeres que tienen mayor nivel educativo disponen de más y mejores oportunidades de empleo en comparación con las que tienen menos años de escolaridad, además sus tasas de participación y de ocupación son significativamente mayores, así como lo es su presencia en las ocupaciones formales de mejor categoría.

\section{Gráfico №2 PEA femenina por hijos menores de 7} años, años 2011 y 2015

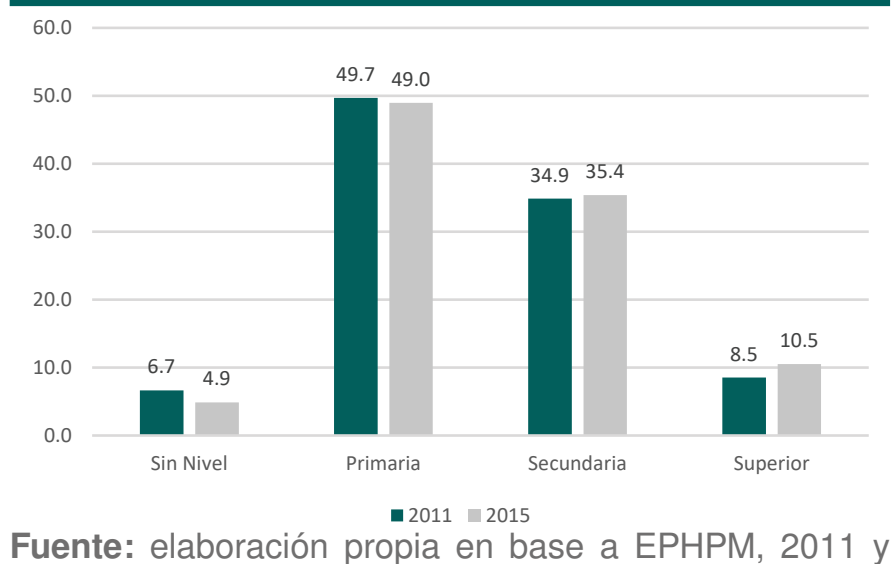
2015, INE.

\subsubsection{PEA femenina según estado civil}

Para la mujer el estado civil puede ser una condicionante importante al momento de ingresar al mercado laboral, lo tradicional es que la mujer al casarse debe encargarse exclusivamente de la producción doméstica, quedándose en el hogar apoyando a su pareja. Esta situación hoy día está cambiando en casi todas las sociedades, pero aún los trabajos empíricos sobre el tema muestran una incidencia negativa del matrimonio sobre la participación de la mujer en el mercado de trabajo (Ferrada, 2010 p. 75).

Se observa que en 2011 el porcentaje de la PEA femenina casada era de $22.4 \%$, para el 2015 ésta representaba el $21.4 \%$, la categoría en unión libre para 2011 era de $20.3 \%$ y para el año 2015 era de $22.0 \%$, es evidente que las mujeres casadas y las mujeres en unión libre, representan aproximadamente el $45 \%$ de la PEA en cada uno de los años de estudio. Ahora bien, al comparar esta categoría "mujeres con pareja" y "mujeres solteras" se observa que las mujeres solteras por sí solas, alcanzan $46.8 \%$ en 2011 ; 48.8\% en 2015 (Gráfico №3).

\section{Gráfico №3 PEA femenina según estado civil,} años 2011 y 2015

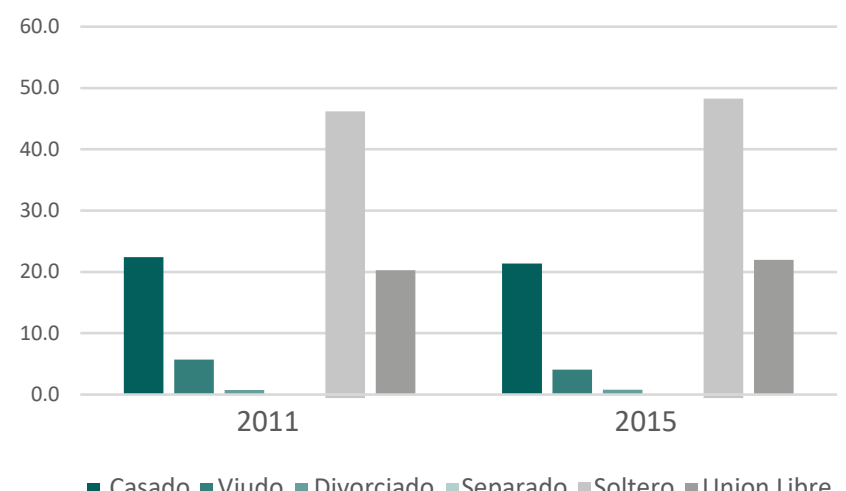

Fuente: elaboración propia en base a EPHPM, 2011 y 2015, INE.

Es evidente las ventajas que tienen las mujeres solteras para competir en el mercado laboral respecto a las mujeres con pareja, muchas empresas prefieren contratar mujeres solteras, convirtiéndose este fenómeno en discriminación principalmente para las mujeres con hijos menores.

Entre estas tres categorías (casadas, unión libre y solteras) se alcanza el $90 \%$ de la PEA femenina, el otro $10 \%$ está distribuido entre las categorías: viuda en 2011 fue de $5.7 \%$; mientras que para 2015 era de $4.1 \%$; las categorías de separado alcanzan valores bajos en ambos años, $4.1 \%$ en 2011 y $3.0 \%$, en 2015, y la categoría de divorciado es de $0.8 \%$ para cada año.

\subsubsection{PEA femenina según área de residencia}

Las diferencias en las condiciones laborales según el área de residencia urbano/rural representan un importante desafío para el país. En 2011 del total 
de la PEA femenina el $59.4 \%$ se encuentra en el área urbana y el $40.6 \%$ en el área rural; estos porcentajes para 2015 representan el $60 \%$ y $40 \%$ respectivamente.

En el área urbana la PEA femenina ocupada en 2011 representó $55.3 \%$ y para el área rural $39.3 \%$; estos porcentajes para 2015 registran $54.4 \%$ y $35.7 \%$, respectivamente (Gráfico $\mathrm{N}^{\circ} 4$ ). Existe un marcado predominio de la PEA femenina ocupada en el área urbana; en 5 años la PEA femenina ocupada decreció $0.9 \mathrm{pp}$ en el área urbana, mientras que para el área rural fue de 3.6 pp en ese mismo período de tiempo; lo que se traduce en PEA femenina desocupada.

La desocupación para la PEA femenina, representa valores bajos en $2011,4.1 \%$ urbano y $1.3 \%$ rural; mientras que para 2015 estos porcentajes son de $5.7 \%$ urbano y $4.3 \%$ rural (Gráfico $N^{\circ} 4$ ).

Gráfico №4 PEA femenina según área urbano/ rural, años 2011 y 2015

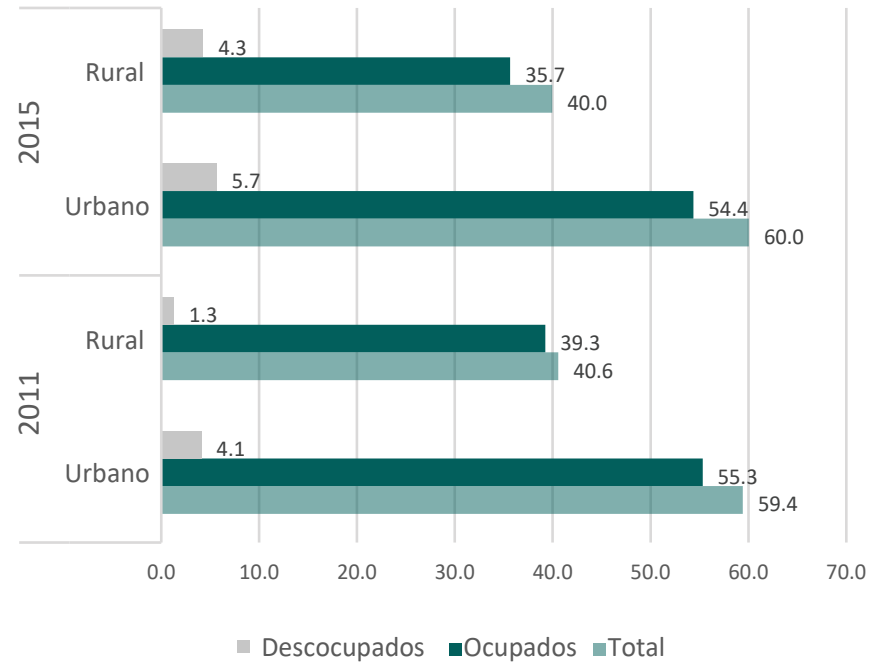

Fuente: elaboración propia en base a EPHPM, 2011 y 2015, INE.

\subsubsection{PEA femenina según nivel educativo}

Un aspecto importante a tener en cuenta en el análisis de la participación laboral está relacionado con los niveles educativos alcanzados por la población que conforma la oferta de fuerza de trabajo.

En el Gráfico N $\$ 5$ los resultados obtenidos muestran que en ambos años, entre las mujeres predomina la educación primaria, $44.8 \%$ en 2011 y $43.0 \%$ en 2015 , lo cual en el contexto de la inserción laboral es negativo, esto conlleva a insertarse en trabajos de baja remuneración, principalmente, las actividades de servicio, el comercio y la manufactura. Según la Teoría del Capital Humano, este fenómeno ocurre en los países con menores niveles de desarrollo, donde bajas tasas de asistencia escolar están relacionadas con mayores niveles de participación laboral, especialmente observadas en poblaciones jóvenes (Elizaga y Mellón, 1971, p. 75); esta situación varía en los países más desarrollados, en los cuales se observa que un mayor nivel en la participación laboral puede estar asociado a niveles educativos más altos.

Los datos muestran que en 2011 las mujeres que tenían secundaria representan $29.6 \%$ y $28.4 \% 2015$, pero al analizar la educación superior se observa que la tendencia de mujeres en el proceso de acumulación de capital humano es positiva para este nivel, el cual para 2011 era $10.5 \%$; mientras que para $2015,11.1 \%$, un incremento de 0.6 puntos porcentuales; en el caso de las mujeres sin educación en 4 años se observa una disminución de $2.3 \%$.

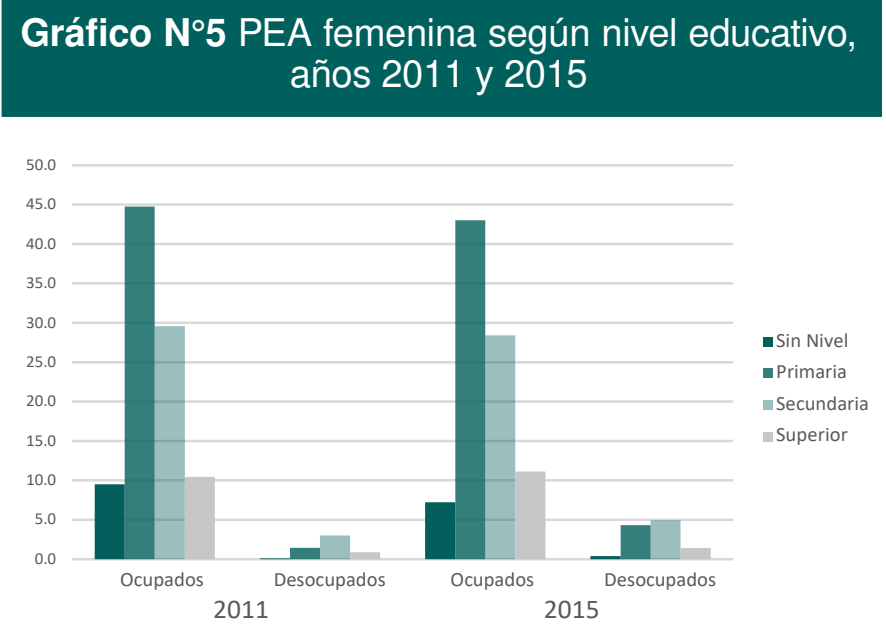

Fuente: elaboración propia en base a EPHPM, 2011 y 2015, INE.

La PEA femenina desocupada para 2011 representa el $5.4 \%$ (62,420 mujeres), y para el año 2015 representa el $10.0 \%$ (150,591 mujeres). La desocupación en el país, alcanza niveles bajos, en general, pero se observa un incremento en la desocupación del $6.0 \%$ de un año a otro, es decir que en 2011 había menos mujeres desocupadas respecto a 2015.

\subsection{Caracterización de la PEA femenina ocupa- da según variables económicas}

En este apartado se hace una caracterización de las mujeres ocupadas y se analizan las variables económicas que visibilizan la participación de la mujer en el mercado laboral como ser: tasa de participación, categoría ocupacional, rama de ocupación y el ingreso por trabajo de las mujeres ocupadas. 
3.2.1. Tasa de participación femenina por nivel educativo, años 2011 y 2015

La tasa de participación femenina por nivel educativo; es un indicador que ayuda a examinar la relación entre escolaridad y actividad de la fuerza de trabajo, comparando las tasas de participación por cada uno de los niveles educativos.

De manera general se puede decir que la tasa de participación femenina muestra una tendencia creciente, conforme aumenta el nivel educativo de las mujeres hondureñas.

La participación de las mujeres que cuentan con nivel educativo superior refleja el porcentaje más alto: 59\% (2011) 64.9\% (2015); en cambio las mujeres que no cuentan con ninguna instrucción alcanzan valores de $27.2 \%$ (2011) y $34.0 \%$ (2015) (Gráfico $\mathrm{N}^{\circ} 6$ ). En el contexto de los datos es evidente la relación directa entre la educación y la participación laboral, tal como lo define la Teoría del Capital Humano, la cual plantea que la inversión en incrementar las capacidades en la población es determinante para acceder a un mercado de trabajo igualitario en la población.

La relación entre educación primaria y secundaria presenta un incremento de aproximadamente $8 \%$ entre un nivel y otro en 2011; mientras que para 2015 este incremento es del 10\% aproximadamente (Gráfico N6).

Gráfico N6 Tasa de participación femenina por nivel educativo, años 2011 y 2015

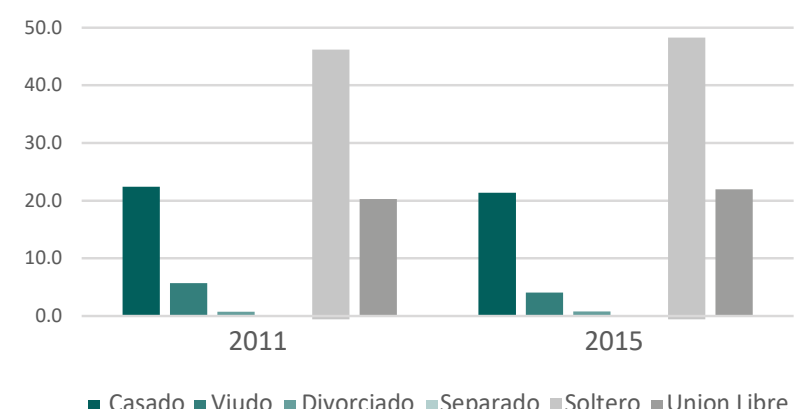

Fuente: elaboración propia en base a EPHPM, 2011 y 2015, INE.

En resumen, niveles educativos altos constituyen un determinante favorable para la participación de la mujer en el mercado laboral; y aumenta la probabilidad de participación de ésta en el mercado de tra- bajo, además la presencia de una relación positiva entre nivel educativo y el ingreso, lo que se convierte en un factor determinante al momento de obtener mejores empleos.

\subsubsection{PEA femenina ocupada, según categoría ocupacional}

La distribución de la PEA femenina por categorías de trabajadores es el reflejo del grado de desarrollo socioeconómico de un país o región. Honduras es un país en proceso de desarrollo, con una especialización económica en el sector primario, la PEA se concentra básicamente en las categorías de "trabajadores por cuenta propia" y "empleado privado".

En Honduras, la categoría cuenta propia alcanza 44.2\% en 2011 y $44.5 \%$ 2015, reflejando un incremento de 0.3 pp en 5 años. Las mujeres en la categoría empleo privado representan el $26.8 \%$ y $25.8 \%$ para cada año respectivamente. Por su parte el empleo público en 2011 representa $9.9 \%$ y para 2015 era de $8.7 \%$, lo que representa una disminución de $1.2 \mathrm{pp}$ en cinco años. El empleo doméstico para 2011 era $7.6 \%$ y en 2015 de $6.8 \%$; mientras que el trabajo no remunerado ascendía a $11.5 \% 2011$ y $14.2 \% 2015$; el trabajo doméstico no remunerado es llevado a cabo por mujeres principalmente mediante labores de cuidados y atención a niños y adultos mayores (Cuadro $N^{\circ} 1$ ).

Al analizar la categoría ocupacional en 2011 para los 4 dominios de estudio de la encuesta se observa que en el Distrito Central 35.7\% se insertaron en empleo privado, $32.1 \%$ trabajaban por cuenta propia; el empleo público representa $16.6 \%$, empleo doméstico $7.6 \%$ y trabajador no remunerado $8.1 \%$. Para 2015 estos porcentajes son $36 \%$; $32 \%$; $15.8 \%$; $7.7 \%$ y $8.5 \%$ respectivamente. En el contexto de San Pedro Sula en estas mismas categorías presenta porcentajes: $45.9 \%$; $32.4 \%$; 7.9\%; 7\%; 6.8\% y para 2015 dichos porcentajes son: 46.1\%; 32.6\%; 5.4\%; 5.9\%; 10\%. El empleo privado tiene su prevalencia en San Pedro Sula y el Distrito Central; estos municipios son los que tienen el mayor crecimiento económico en el país, sobre todo San Pedro Sula; que es donde se concentra la mayor parte de la actividad industrial de Honduras.

En el área rural prevalece el empleo por cuenta propia $54.4 \%$ en 2011 y $55.1 \%$ en 2015 ; la PEA femenina ocupada en esa zona generalmente se dedica a las actividades agrícolas, ganaderas y a la cría de animales domésticos; el trabajador no remunerado en 2011 representa $14.8 \%$ y $20.1 \%$ en 2015 ; mientras que el empleo privado alcanza valores de $18.2 \% 2011$ y $15.1 \%$ en 2015. 
Cuadro №1 PEA femenina ocupada por categoría ocupacional, años 2011 y 2015

\begin{tabular}{|c|c|c|c|c|c|c|c|}
\hline \multirow{3}{*}{ Año } & \multirow{3}{*}{$\begin{array}{l}\text { Dominio } \\
\text { Estudio }\end{array}$} & \multirow[b]{2}{*}{ Total } & \multicolumn{5}{|c|}{ Categoría Ocupacional } \\
\hline & & & $\begin{array}{l}\text { Empleado } \\
\text { Público }\end{array}$ & $\begin{array}{c}\text { Empleado } \\
\text { Privado }\end{array}$ & $\begin{array}{l}\text { Empleada } \\
\text { Doméstica }\end{array}$ & $\begin{array}{l}\text { Cuenta } \\
\text { Propia }\end{array}$ & $\begin{array}{c}\text { Trabajador } \\
\text { no } \\
\text { Remunerado }\end{array}$ \\
\hline & & /1 & 12 & 12 & 12 & 12 & 12 \\
\hline \multirow{5}{*}{2011} & Total & 100.0 & 9.9 & 26.8 & 7.6 & 44.2 & 11.5 \\
\hline & Distrito Central & 17.4 & 16.6 & 35.7 & 7.6 & 32.1 & 8.1 \\
\hline & $\begin{array}{l}\text { San Pedro } \\
\text { Sula }\end{array}$ & 9.8 & 7.9 & 45.9 & 7.0 & 32.4 & 6.8 \\
\hline & Resto Urbano & 31.3 & 12.5 & 27.3 & 8.5 & 41.3 & 10.4 \\
\hline & Rural & 41.5 & 5.4 & 18.2 & 7.1 & 54.4 & 14.8 \\
\hline \multirow{5}{*}{2015} & Total & 100.0 & 8.7 & 25.8 & 6.8 & 44.5 & 14.2 \\
\hline & Distrito Central & 16.5 & 15.8 & 36.0 & 7.7 & 32.0 & 8.5 \\
\hline & $\begin{array}{l}\text { San Pedro } \\
\text { Sula }\end{array}$ & 7.1 & 5.4 & 46.1 & 5.9 & 32.6 & 10.0 \\
\hline & Resto Urbano & 36.8 & 11.0 & 28.9 & 7.9 & 41.0 & 11.3 \\
\hline & Rural & 39.6 & 4.2 & 15.1 & 5.5 & 55.1 & 20.1 \\
\hline \multicolumn{8}{|c|}{$\% / 1$ por columna } \\
\hline \multicolumn{8}{|c|}{$\% / 2$ por fila } \\
\hline
\end{tabular}

Fuente: elaboración propia en base a EPHPM, 2011 y 2015, INE.

En el resto urbano del país, los valores más significativos son: cuenta propia $41.3 \%(2011)$ y $41 \%$ (2015); luego está el empleo privado $27.3 \%$ (2011) y $28.9 \%$ (2015) (Cuadro Nº1).

En general, alrededor del $42.8 \%$ de la población femenina ocupada se encuentra desarrollando actividades como asalariada, un $44.3 \%$ son cuenta propia y trabajador no remunerado $12.8 \%$; que para el caso de 2015 tuvo un incremento de $2.7 \%$, respecto a 2011 (Cuadro N¹).

\subsubsection{PEA ocupada femenina según rama de acti- vidad, año 2011 y 2015}

La rama de actividad refleja la evolución de la economía de un país, permitiendo la generación de riqueza, mediante la extracción, transformación y distribución de los recursos naturales o de la prestación de algún tipo de servicio.

La rama de actividad de la PEA femenina ocupada se encuentra principalmente, en la agricultura, industria manufacturera, comercio por mayor y menor; y en la rama de los servicios. Se observa que en su mayoría se insertan en el comercio por mayor y menor $35.9 \%$ en ambos años. En segundo lugar, la rama de los servicios con $29.2 \%$ en 2011 y $27.8 \%$ 2015 , la industria manufacturera observa el tercer lugar con $18.8 \%$ y $18.4 \%$ respectivamente, y luego le sigue la agricultura $11.3 \%$ en 2011 y $12.5 \%$ en 2015 (Gráfico N7).
El comercio, y los servicios siguen siendo los grandes empleadores de la población femenina en ambos años; las ocupaciones en estos sectores tienden a ser de menor calidad, con bajas remuneraciones, pocas posibilidades de promoción, precarias condiciones laborales y escasa estabilidad, demostrando fuertes implicaciones de discriminación.

Las cifras muestran que aproximadamente el $65 \%$ de las mujeres ocupadas se encuentran realizando actividades de servicios y el comercio, lo que no garantiza salarios que satisfagan las necesidades de las mujeres hondureñas ni de sus familias, generalmente se emplean como: empleadas domésticas, lavanderas, vendedoras, en hoteles, restaurantes, peluquerías, panaderías, tortillerías, etc.

\section{Gráfico $N^{0} 7$ PEA femenina ocupada por rama de} actividad, años 2011 y 2015

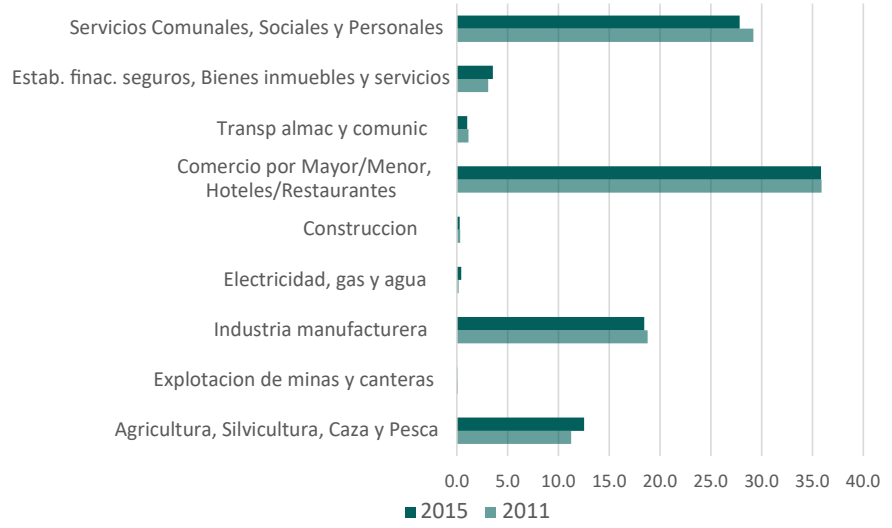

Fuente: elaboración propia en base a EPHPM, 2011 y 2015, INE. 


\subsubsection{Influencia del nivel educativo en los ingresos de las mujeres ocupadas}

La educación es uno de los principales determinantes del nivel de vida de la población. Muchos estudios tratan de explicar la relación que existe entre el nivel educativo y los ingresos de una persona; la inversión en educación o especialización traerá crecimiento y beneficios al individuo contribuyendo a su máximo desarrollo y bienestar, tal como lo manifiesta la teoría del capital humano (Becker, 1987, p. 2).

En el Gráfico $\mathrm{N}^{\circ} 8$, se presenta el efecto de la educación en el nivel educativo de las mujeres ocupadas en los años 2011 y 2015, es decir, ¿Cuál es el ingreso de las mujeres según el nivel educativo?

Hay una relación directa entre el nivel educativo y los ingresos de las mujeres, se dice que a mayor nivel educativo mayores ingresos. Se observa que los mayores ingresos promedio los obtienen las mujeres con nivel de educación superior L. 11,292 en 2011 y L. 14,943 en 2015. Si se hace una relación entre los ingresos percibidos con el siguiente nivel (secundaria), existe una diferencia de aproximadamente el doble a favor de las mujeres con nivel superior; debido a que las mujeres que obtuvieron nivel educativo de secundaria tienen ingresos promedios de $\mathrm{L}$. 5,393 en 2011 y L. 6,530 en 2015.

Los ingresos de las mujeres que solo tenían educación primaria eran L. 2,894 para 2011 y L. 3,713 en 2015; mientras que las mujeres sin ningún nivel de educación obtuvieron los ingresos promedio más bajos L. 1,844 en 2011 y L. 2,520 en 2015; la diferencia de ingresos entre las mujeres que obtuvieron primaria respecto a las que no tenían ningún nivel es del $50 \%$.

\section{Gráfico N`8 Mujeres ocupadas por ingreso} promedio según nivel educativo, años 2011 y 2015

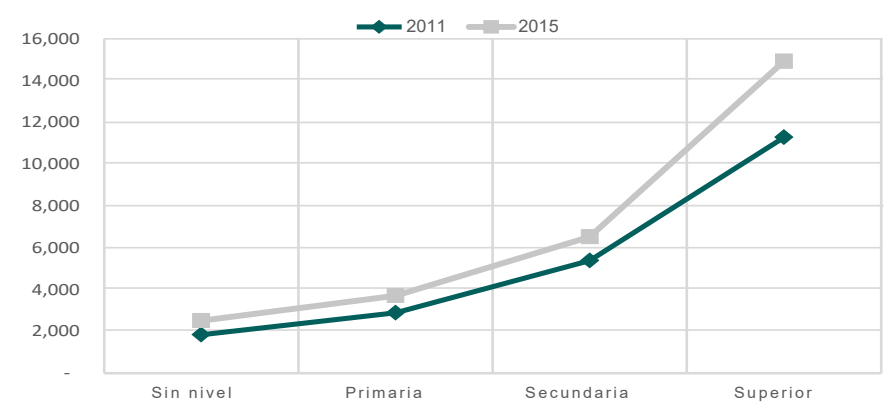

Fuente: elaboración propia en base a EPHPM, 2011 y 2015, INE.
De esta manera se evidencia que el nivel educativo contribuye al desarrollo alcanzado por la mujer, lo que conlleva al logro de bienestar y satisfacción personal y de su familia, mediante la obtención de ingresos suficientes para sí misma y su entorno. Es sabido que la educación es un determinante elemental de la posición de las personas en el mercado laboral y los trabajadores más educados tienden a acceder a puestos de trabajo mejor pagados.

\subsection{Estimación y desarrollo de Modelo Probit Multivariado}

En este apartado se presenta la correlación entre variables y el peso de estas respecto a la variable independiente en su conjunto, y con esta estimación revisar si existen cambios de tendencia (negativa o positiva) y en la magnitud de los coeficientes de determinación de dichas variables.

Modelo resultante 2011/2015: probit trab_1 tothijos casado, rurur2, jefemuj, edu2, edu3, edu4 [w=pondera] if sexo $==2$ \& inrange(edad, 10,64), robust.

Para el año 2011; el número de observaciones fueron 765,612; mientras que para el año 2015 las observaciones fueron 873,044 .

La variable tothijos (hijos menores de 7 años); casado y área rural, resultaron con signo negativo en ambos años; esto quiere decir que la probabilidad de participación disminuye si la mujer está casada o en pareja, o si reside en el área rural o tiene hijos menores de 7 años. Los coeficientes de determinación variaron en su estimación de un año a otro, por ejemplo, la variable tothijos, es de $-8 \%$ a un $-9 \%$ : 2011/2015; es decir que la probabilidad de participación disminuye para la mujer si tiene hijos menores de siete años.

Según el modelo se observa una tendencia negativa de la variable casado: $-47.04 \%$ y $-47.09 \%$ 2011/2015; lo que indica que la probabilidad de la mujer de insertarse en el mercado laboral es negativa si está casada o en pareja.

Según el modelo la variable área de residencia rural refleja una tendencia negativa: $-29 \%$ y $-26 \%$ en 2011/2015; evidenciando que las mujeres que viven en el área rural tienen menor probabilidad de insertarse en el mercado de trabajo, influenciado este fenómeno por el bajo nivel educativo. 
La tendencia de la variable jefa de hogar es positiva 49.54\% (2011), 29.28\% (2015); es decir, la probabilidad de que la mujer tenga un empleo es positiva si es jefe de hogar, obviamente la mujer al ser el único sostén de su hogar se ve forzada a trabajar en condiciones de inequidad aceptando cualquier trabajo que le ofrezcan o en todo caso laborando en el mercado informal.

Para la variable educación la tendencia de los coeficientes de determinación son positivos cuando la mujer tiene educación primaria 28\% (2011), y 8\% (2015); de igual manera es el comportamiento si la mujer tiene nivel de educación secundaria 47\% (2011) y 25\% (2015), y para el nivel superior; el modelo multivariado representa una mayor estimación en magnitud $81 \%$ y $68 \%$ respectivamente (Cuadro $\mathrm{N}^{\circ} 2$ ).

La prueba de clasificación del modelo (estatclass) mide la correcta clasificación o predicción de los casos en el modelo, y este para 2011 fue de $68.49 \%$ y para 2015 de $64.80 \%$; se estima que el modelo arriba del $60 \%$, está prediciendo correctamente.

La prueba del efecto marginal (mfx): proporciona el efecto marginal de las variables, es decir el efecto que se produce en la variable independiente cuando hay una variación en las variables independientes, en el modelo multivariado estimado para 2011 fue de 34.21\%; para 2015 de 42.24\%, (Cuadro N²).

En ambos años, el nivel educativo tiene un fuerte y positivo impacto en la probabilidad de participación laboral de la mujer. Así en el año 2011, una mujer que tiene educación superior completa tiene una probabilidad de participar $50 \%$ y $60 \%$ más que una mujer con educación primaria según el modelo.

La hipótesis propuesta en el estudio fue: Los factores que inciden en la participación laboral de la mujer hondureña son, el número de hijos (<7 años), el estado civil (casado), el área de residencia (rural), jefatura de hogar y el nivel educativo: por lo tanto se puede concluir que no existe suficiente evidencia para rechazar la hipótesis propuesta; pues queda evidenciado que el número de hijos menores, el estado civil de casado y el área de residencia rural tienen un comportamiento negativo en la participación de la mujer en el mercado laboral; mientras que el nivel educativo y la jefatura de hogar femenina influyen positivamente en la participación de la mujer en el mercado de trabajo y a medida que aumenta el nivel educativo también aumentan las probabilidades de participación como se muestra en los resultados del modelo.

\section{Cuadro $N^{\circ} 2$ Resultado de las estimaciones del Modelo Probit Multivariado}

\begin{tabular}{|l|r|r|r|r|}
\hline \multirow{2}{*}{ Variables } & \multicolumn{2}{|c|}{2011} & \multicolumn{2}{c|}{2015} \\
\cline { 2 - 5 } & Coef Consolidado & \multicolumn{1}{c|}{ Valor P } & Coef Consolidado & \multicolumn{1}{c|}{ Valor P } \\
\hline tothijos & -0.08858 & 0.000 & -0.0949076 & 0.000 \\
\hline casado & -0.4705616 & 0.000 & -0.470982 & 0.000 \\
\hline rurur2 & -0.2938094 & 0.000 & -0.2676683 & 0.000 \\
\hline jefemuj & 0.4954369 & 0.000 & 0.2928358 & 0.000 \\
\hline edu2 & 0.2863249 & 0.000 & 0.0845988 & 0.000 \\
\hline edu3 & 0.4771042 & 0.000 & 0.2574706 & 0.000 \\
\hline edu4 & 0.8186222 & 0.000 & 0.6815603 & 0.000 \\
\hline cons & -0.9623938 & 0.000 & -0.4975669 & 0.000 \\
\hline No. Observaciones & 765,612 & & 873,044 & \\
\hline Wald & 83384.05 & & 83615.84 & \\
\hline Mfx & 0.342196 & & 0.42246051 & \\
\hline Correctly classified & $68.49 \%$ & & $64.80 \%$ & \\
\hline
\end{tabular}




\section{Conclusiones}

Las mujeres, a través de los años, han logrado avances significativos, han roto barreras; han incursionado y avanzado paulatinamente en la educación, han logrado incursionar en su participación política y hoy siguen trabajando para lograr la igualdad, inserción y permanencia en el mercado de trabajo sesgado al sexo masculino.

El nivel educativo predominante entre la PEA femenina hondureña en los dos años de estudio es el de educación primaria. La educación de las mujeres incide en la calidad de vida, su situación socioeconómica y de manera importante en sus actitudes y prácticas relacionadas con su quehacer, con la salud y el comportamiento reproductivo, lo cual se traduce en una disminución en las tasas de fecundidad.

Se evidenció que los ingresos de la población femenina muestran una tendencia creciente, conforme aumenta el nivel de instrucción en las mujeres hondureñas; lo que indica que hay una relación directa entre la educación, los ingresos y la participación laboral femenina. Se puede concluir que los niveles educativos son relevantes para propiciar una mayor participación laboral y mayores remuneraciones para las mujeres.

En el análisis de los modelos desarrollados se identifica que tener hijos menores de edad, disminuye la probabilidad de que la mujer se inserte en el mercado laboral, el modelo resultó con tendencia negativa, sobre el nivel educativo, resultó ser una variable muy positiva, el nivel educativo, proporciona cierta mejoría económica, pero ésta no es suficiente ya que existe un porcentaje de mujeres que a pesar de tener niveles educativos superiores se encuentran en situación de subempleo.

\section{Referencias bibliográficas}

- Aguilar-Barceló, J. G., A. B,Mungaray-Moctezuma, M. Ramirez-Urquidy, 2014, "Género y autoempleo en México durante la recesión de 20082009: un estudio de flujos laborales", en Papeles de Población, vol. 20, núm. 80, Baja California, México.

- Anker, R. (1997). Segregación Profesional entre hombres y mujeres. Revista Internacional del Trabajo, Pág.343-370, OIT Ginebra.
- Gary Becker, (1983). Inversión en capital humano e ingresos, en Luis de Tohaira. El mercado de trabajo: teorías y aplicaciones. Alianza Universidad. España.

- Castillo Laborde, C. (2005). Determinantes Económicos de la Fecundidad de Corto Plazo en Chile. Santiago de Chile: Pontificia Universidad Católica de Chile.

- CELADE (1996), Boletín Demográfico de América Latina, pág. 29), Santiago de Chile.

- DEMOMUJER, UNAH-MDD, (2012), Volúmen 1, Mercado Laboral: 10 años de historia de las mujeres hondureñas, Litografía López, S. de R. L. Tegucigalpa, MDC, Honduras.

- Euwals, Rob (2001), "Female Labour Supply, Flexibility of Working Hours, and Job Mobility", the Economic Journal, (111) 2001, Oxford University Press.

- García, C. (2013). Descenso de la Fecundidad, participación laboral de la mujer y reducción de la pobreza en Colombia 1990-2010.Bogotá: Gobierno de Colombia, UNFPA.

- Gelbach, J. (2002) "Public Schooling for Young Children and Labor Supply", Economic Review, 2002, 92(1), pp. 307-22, University of Maryland.

- Gobierno de Chile. (2002). Normas Nacionales sobre regulación de la fertilidad, Ministerio de Salud, Santiago de Chile.

- Instituto Empresa y Humanismo, №59, junio 2005, pp. 15-17 Universidad de Navarra Pamplona España. 